\title{
KATHARINA MIDDELL
}

\section{HUGENOTTEN IN KURSACHSEN EINWANDERUNG UND INTEGRATION}

Welche Kriterien berechtigen dazu, in der Frühen Neuzeit von Einwanderungspolitik zu sprechen? Die Emigration der Hugenotten aus Frankreich, eine der größten Völkerwanderungen der Frühen Neuzeit, bot wohl erstmals die Möglichkeit, ein zielgerichtetes Programm für ihre Aufnahme zu entwickeln, das sich durch ein erkennbares $\mathrm{Ma} \beta$ an Planung, Kontinuität und Konsequenz in der Durchsetzung als Politik bezeichnen ließe. In jedem Fall gilt es, bei der Prüfung von Einwanderungspolitik die Institutionen des jeweiligen Landes zu befragen, ihre Kompetenzen und ihre langfristigen Ziele vorzustellen. Integration als langfristiger Proze $B$ kann kaum auf dem politischen Verordnungsweg erreicht werden. Sie setzt Offenheit und Anpassungsbereitschaft sowohl bei der Migrantengruppe als auch in der Gesellschaft des Aufnahmelandes voraus. Integrationsprozesse verlaufen eher an der Basis und im Alltag, es gilt daher, diejenigen gesellschaftlichen Gruppen zu betrachten, in denen die Einwanderer agierten.

Für Kursachsen sind zuerst die Vorbedingungen im Territorium selbst zu schildern, aus denen sich die Intentionen der Kurfürsten und der Landesbehörden ergaben. Es wird zu sehen sein, daß es, abgesehen von sporadischen Ansätzen, keine Einwanderungspolitik im oben genannten Sinne gab und daß Ansiedlungsversuche Friedrich Augusts I. zum Scheitern verurteilt waren. Dann wäre zu begründen, warum Sachsen bei der Aufnahme der Hugenotten eine so nachrangige Bedeutung bekam. Es wird gezeigt, daß sich die Gestaltung der Niederlassungsbedingungen für Hugenotten und das Verhältnis zwischen Zuwanderern und Aufnahmegesellschaft in Kursachsen von allen anderen deutschen Aufnahmeländern unterscheiden. Im zweiten Teil wird vorrangig am Beispiel von Leipzig die Entstehung der reformierten Gemeinde und ihre Besonderheit untersucht. Die verschiedenen Faktoren, die die gesellschaftliche Integration der Hugenotten während des 18. Jahrhunderts bis zur 1811 errungenen Gleichberechtigung beförderten, werden abschließend erörtert. Insgesamt läßt sich nicht bestreiten, da $B$ die hugenottische Migration trotz ihrer anfangs versuchten Verhinderung eine positive Erfahrung mit sich brachte - für die Hugenotten und ihre Nachfahren ebenso wie für die Aufnahmegesellschaft, nachdem im Verlauf von drei bis vier Generationen die anfängliche Distanz und Fremdheit abgebaut worden war. 


\section{Vorbedingungen}

Seit der Reformation war Sachsen ein lutherisches Land, die Confessio Augustana invariata die herrschende Landesreligion; die Episode des kalvinistischen Kurfürsten Christian I. führte Ende des 16. Jahrhunderts zur Erhärtung der lutherischen Orthodoxie. Die Alleinherrschaft der lutherischen Lehre erzwang, daß alle Anhänger einer fremden Konfession nur »mindere« Bürger sein konnten, die zwar als Einwohner ein Bleiberecht genießen durften, aber von städtischen Bürgerrechten und allem, was mit dem Bürgerrecht verbunden war, ausgeschlossen blieben.

Der Landesherr war wie überall in den protestantischen Territorien des Alten Reiches das Oberhaupt der Landeskirche. Das Geheime Consilium als zentrale Verwaltungsinstitution bzw. nach 1706 unter August dem Starken das Geheime Kabinett als oberste Zentralbehörde hatte auch für den Schutz des Augsburgschen Bekenntnisses zu sorgen'. Vor allem aber das Oberkonsistorium in Dresden als kirchliche Oberbehörde widmete sich der Reinhaltung des orthodoxen Luthertums. Die reformiertenfeindliche Haltung der sächsischen Geistlichkeit ${ }^{2}$ zeigte sich in allen einschlägigen Berichten und Gutachten an den Landesherrn: Da wird die Schädlichkeit der Kalvinisten beschworen, ihre irrige und falsche Lehre, die drohende Verführung der Mit-Christen und Unterthanen. Die Fundamentalverfassung, in der die lutherische Konfession fest verankert war, dürfe schon gar nicht der Landesherr selbst verletzen, und aus rein weltlichen Gründen, also auch zur wirtschaftlichen Förderung des Landes, verböte sich die Aufnahme Falschgläubiger grundsätzlich. Die Kurfürsten Johann Georg III. (1680-1691) und Johann Georg IV. (1691-1694) hatten noch stets die konfessionellen Bedenken des Geheimen Rates und der Stände geteilt und die vorsichtig vorgetragenen Bitten der Brandenburger Kurfürsten abgeschlagen. So wurde Johann Georg IV. wiederholt von Friedrich III. angehalten, sich für die aus Frankreich der Religion halber vertriebenen Reformirten einzusetzen und denen, die sich in Kursachsen mittlerweile aufhielten, wenigstens eine bedingte Religionsfreiheit einzuräumen. Doch Johann Georg verwehrte diese Konzession im Einvernehmen mit dem Oberkonsistorium, das schon im November 1691 und wiederholt im Februar 1692 Bedenken vortrug, nicht nur weil die Reformierten die Seeligkeit nicht erhoffen könnten, sondern weil zudem die Päbstlichen Clerici sich dieses Vortheils gleichfalls bedienen würden ${ }^{3}$. Es ging in Kursachsen bis zum Anfang des 18. Jahrhunderts, als

${ }^{1}$ Über Verwaltungsaufbau und Behörden in Kursachsen unter Friedrich August I. siehe Reiner GROSs, Geschichte Sachsens, Leipzig 2001, S. 131-134.

${ }^{2}$ Rudolf KÖTZSCHKE, Hellmut KRETZSCHMAR, Sächsische Geschichte, Frankfurt a. M. ${ }^{2} 1965$, S. 257.

${ }^{3}$ Sächsisches Hauptstaatsarchiv Dresden (= SHStAD), Loc. 10 333, Bl. 37-38, 3./13. November 1691; Bl. 40-44, 15. Februar 1692; Bl. 49-50, 11. Dezember 1694. 
Friedrich August I. (1694-1733) für die Leipziger Reformierten die Erlaubnis zur Ausübung ihres Gottesdienstes durchsetzte, mitnichten um eine geförderte, auch nicht um eine wohlwollend gebilligte Einwanderung von Refugiés, mochte der Brandenburger seinen vielgeliebten Vetter und Bruder noch so sehr bedrängen.

Wie für andere deutsche Territorien stand die zweite Hälfte des 17. Jahrhunderts auch in Kursachsen unter dem Zeichen des wirtschaftlichen Wiederaufbaus nach dem Dreißigjährigen Krieg ${ }^{4}$. Das Land zählte zu den am stärksten heimgesuchten Gebieten des Reiches ${ }^{5}$. Die Motivation der protestantischen deutschen Landesfürsten, die fliehenden Hugenotten aufzunehmen, wurzelte gerade in der Absicht, die Kriegsschäden zu beseitigen, wüste Landstriche zu peuplieren und die Wirtschaft wieder anzukurbeln. Für Kursachsen gewannen - lange vor dem französischen Revokationsedikt von 1685 - die böhmischen Protestanten besondere Bedeutung. Vertrieben durch die Rekatholisierungspolitik des habsburgischen Kaisers Ferdinand II., wanderten aus dem Nachbarland zwischen 1620 und 1660 rund 150000 Böhmen ein ${ }^{6}$ - zehn bis 15 Prozent der damaligen Einwohnerzahl in Kursachsen. Der rasche Aufschwung der Gewerbetätigkeit, besonders im südlichen Landesteil, war wesentlich den böhmischen Zuwanderern zu verdanken.

Die Ansiedlung der böhmischen Exulanten, treuer Lutheraner, wurde von den sächsischen Kurfürsten im allgemeinen großzügig gehandhabt, wenngleich es keine gezielte Kolonisationspolitik gab. Große zusammenhängende Brachflächen zur Peuplierung gab es ohnehin nicht mehr ${ }^{7}$. Das muß im Unterschied zu Brandenburg betont werden, wo die Verwüstung der ländlichen Gebiete eine Ansiedlung der Hugenotten geradezu herausforderte ${ }^{8}$. Anfang der neunziger Jahre des 17. Jahrhunderts waren die ökonomischen Auswirkungen des Dreißigjährigen Krieges in Sachsen weitgehend überwunden?.

Zugespitzt mag das aber auch bedeuten: Eine gezielte Anwerbung der französischen Kalvinisten war - unabhängig von Glaubensfragen - wirtschaftlich nicht zwingend notwendig. Allerdings beklagten zeitgenössische Merkantilisten wie der königlich-polnische und kursächsische Hof- und Kommerzienrat Marperger gerade im Vergleich $\mathrm{zu}$ Brandenburg-Preußen das versehene Nichteinnehmen der Refugierten Frantzosen, besonders die damit entgangene[n] Capitalisten ${ }^{10}$.

\footnotetext{
${ }^{4}$ Heinz DuCHHARDT, Das Zeitalter des Absolutismus, München 1989 (Oldenbourg GrundriB der Geschichte, 11), S. 18-21.

${ }^{5}$ KOTZSCHKE, KRETZSCHMAR, Sächsische Geschichte (wie Anm. 2), S. 254.

${ }^{6}$ Ibid., S. 244f.; Gross, Geschichte Sachsens (wie Anm. 1), S. $102 f$.

${ }^{7}$ Ibid., S. 103.

${ }^{8}$ Ingrid MITTENZWEI, Erika HERZFELD, Brandenburg-Preußen 1648 bis 1789. Das Zeitalter des Absolutismus in Text und Bild, Berlin 1987, S. 145-148.

${ }^{9}$ Gross, Geschichte Sachsens (wie Anm. 1), S. 105.

${ }^{10}$ Paul Jacob MARPERGER, Abriß der Commercien und Manufacturen des Churfüstenthum Sachsens und seiner incorporirten Länder, Dresden, Leipzig 1718, S. 17.
} 
Zur Reinhaltung der evangelisch-lutherischen Landesreligion und der relativen wirtschaftlichen Stärke Kursachsens kam ein dritter Punkt hinzu, der die Aufnahme französischer Glaubensflüchtlinge behinderte und erschwerte: die nur selten inkonsequente, traditionell kaisertreue Außenpolitik der sächsischen Landesherren seit Kurfürst Moritz ${ }^{11}$. Da Sachsen seit 1689 wiederum im Reichskrieg gegen Frankreich stand, konnte es auch kein Interesse daran haben, Franzosen ins Land zu holen, auch wenn die Hugenotten >Feinde des Reichsfeindes Ludwig XIV. waren. Kalvinisten sollten in Sachsen nicht heimisch werden ${ }^{12}$.

\section{Ansiedlungsversuche}

Es gab allerdings einige Versuche der gezielten Ansiedlung französischer Glaubensflüchtlinge. Sie zeichneten sich alle dadurch aus, daß es niemals zu offiziellen Rechtsakten wie Privilegien oder ähnlichem kam. Diese Versuche beginnen 1694 und fallen damit in die Herrschaftszeit des Kurfürsten Friedrich August I., der als August II. (1697) König von Polen wurde. In seiner Regierungszeit wandelte sich das Verhältnis des Herrschers zur Aufnahme französischer Hugenotten - im Gegensatz zu seinen Vorgängern. Da Friedrich August selbst einen Glaubenswechsel durchmachte, mochte er ein wenig toleranter oder auch religiös indifferent ${ }^{13}$ sein, er hatte ja auch französische kalvinistische Hofmeister und Kammerdiener. Allein durch seine Entscheidung und Unterstützung konnte in Leipzig die reformierte Kirchengemeinde entstehen. Andererseits wuchs durch den Übertritt Friedrich Augusts zum katholischen Glauben die Macht des Geheimen Rates, der seit der Konversion das landesherrliche Kirchenregiment ausübte ${ }^{14}$.

${ }^{11}$ KOTZSCHKE, KRETZSCHMAR, Sächsische Geschichte (wie Anm. 2), S. 243.

${ }^{12}$ Das Verhältnis des Luthertums zum Kalvinismus war im Kernland der Reformation besonders seit der »Zweiten Reformation « problematisch. Vgl. über den »Kalvinistensturm « in Leipzig 1593: Karl CzOK, Der »Calvinistensturm« 1592/93 in Leipzig - seine Hintergründe und bildliche Darstellung, in: Jahrbuch zur Geschichte der Stadt Leipzig 1977, S. 123-144. Ende des 18. Jahrhunderts konnte es bereits abgeklärt heißen, nach dem Kalvinistensturm sei Leipzig in beständigem ruhigem Besitze der mreinen evangelischen Religion « geblieben, alle anderen Religionen und Sekten würden geduldet, »sobald sie dem Staate unschädlich sind«, Johann Gottlieb SCHULZ, Beschreibung der Stadt Leipzig, Leipzig 1784, S. 171; vgl. auch Anm. 33.

${ }^{13}$ Karlheinz BLASCHKE, Der Konfessionswechsel des sächsischen Kurfürsten Friedrich Augusts I. und seine Folgen, in: Sachsen und Polen zwischen 1697 und 1765. Beiträge der wissenschaftlichen Konferenz vom 26. bis 28. Juni 1997 in Dresden, hg. v. Verein für sächsische Landesgeschichte, Dresden 1998, S. 210-222, hier S. 210; KOTZSCHKE, KRETZSCHMAR, Sächsische Geschichte (wie Anm. 2), S. 268.

${ }^{14}$ BLASCHKE, Konfessionswechsel (wie Anm. 13), S. 219. 
Trotz seines zunehmend absolutistischen Gebarens war der Landesherr nicht frei in seiner Entscheidung. Friedrich August mußte sich zwischen den Zwängen der eigenen Ziele, seiner Rolle im Reich als Hüter des Protestantismus Kursachsen hatte den Vorsitz im Corpus Evangelicorum der lutherischen Reichsstände - sowie im Einvernehmen mit dem Geheimen Rat, den Landständen und der Geistlichkeit bewegen. So spielten schon in dem langwierigen und hochgradig umstrittenen Ansinnen, den in Leipzig ansässigen reformierten Kaufleuten die private Religionsausübung zu gestatten, diplomatische Rücksichten eine wesentliche Rolle: In den Bündnisverhandlungen mit England und den Niederlanden im Vorfeld des Nordischen Krieges hatte sich Friedrich August verpflichten müssen, die Reformierten, als Religionsverwandte der beiden Bündnispartner, zuvorkommend zu behandeln ${ }^{15}$. Das Geheime Consilium dagegen versuchte ihm klarzumachen, daß er auf die Erhaltung des Reichs-Directoriums der lutherischen Reichsstände bedacht sein müsse und er sich bei den evangelischen Kurfürsten und Ständen einen großen Haß und wohl gar den Verlust des Directorii erwirken würde, wenn er den Reformierten entgegenkäme ${ }^{16}$.

Trotz der massiven Gegenwehr seiner Räte und der Stände versuchte Friedrich August, im Jahr 1708 in den Städten Torgau, Meißen und Eilenburg Hugenotten anzusiedeln. Besonders das alte Torgau war seit der schwedischen Invasion im Dreißigjährigen Krieg und durch die Pest sehr schwer mitgenommen ${ }^{17}$. Sein Anliegen bestand - durchaus gewöhnlich - darin, zur Aufnahme derer Commercien und Nahrung in den Erblanden [...] die französische und andere Kaufleute, Traficanten und Manufacturiers reformierter Religion in gedachte unsere Lande zu ziehen ${ }^{18}$. Der Torgauer Stadtrat wehrte sich freilich gegen die per Reskript vom 12. Juni 1708 in Aussicht gestellte Ansiedlung von bey nahe [...] vier hundert Persohnen, refugirten französischen Manufacteurs $^{19}$ und fuihrte an erster Stelle nicht die differente Religion an, sondern die bedrohten Versorgungsverhältnisse der Stadt.

Wirtschaftliche Vorteile für Torgau seien nicht absehbar, hieß es da, weil sie [die Refugirten] eigene Becker, Schuster, Schneider, und dergleichen Handwercks-Leuthe hätten und zu allem Überfluß ihr eigenes so genandtes Frantzösisches Bier brauen wollten, wo doch der Haupterwerbszweig der Stadt gerade die Bierbrauerei sei. Zum städtischen Konsum würden die Refugiés also

${ }^{15}$ Die Leipziger Reformierten beschritten unabhängig davon den Petitionsweg an besagte Mächte, in Leipzig vertreten durch den englischen und niederländischen Gesandten. Albrecht KIRCHHOFF, Geschichte der Reformirten Gemeinde in Leipzig von ihrer Begründung bis zur Sicherung ihres Bestandes, 1700-1725, Leipzig 1874, Beilage XVIII.

${ }^{16}$ SHStAD (wie Anm. 3), Loc. 10 333, Bl. 185-189. Über die Lösung des Problems siehe BLASCHKE, Konfessionswechsel (wie Anm. 13), S. $219 f$.

${ }^{17}$ GROSS, Geschichte Sachsens (wie Anm. 1), S. 100.

${ }^{18}$ SHStAD (wie Anm. 3), Loc. 10 333, Bl. 215, Warschau 8. Juni 1713, an Geheimes Konsilium.

${ }^{19}$ Ibid., Bl. 204, Reskript vom 12. Juni 1708 an Kanzler, Vizekanzler und Geheime Räte. 
nichts beitragen, weil sie autark leben wollten. Außerdem sei eine dauerhafte Niederlassung der Franzosen wegen derselben bekandten all zu großen Liebe gegen ihr Vaterlandt ohnehin nicht zu erwarten. Mißachtung der Gesetze, Konflikte mit den übrigen Einwohnern und weitere Probleme wurden ins Feld geführ ${ }^{20}$, zumal die Refugiés sich der ordentlichen Gerichtsbarkeit entziehen wollten - wodurch die übrigen Einwohner schwierig gemacht werden - und eine eigene Obrigkeit und Jurisdiktion anstrebten ${ }^{21}$.

So scheiterte 1708 der landesfürstliche Ansiedlungsplan für Torgau am Widerstand der Bürger und ihrer städtischen Vertreter. Ähnlich erging es den anderen Städten.

Die überwiegend wirtschaftlichen Argumente weisen darauf hin, daß es dem Stadtrat und den Bürgern von Torgau vor allem darum ging, den Zuzug wirtschaftlicher Konkurrenten sowie bedürftiger und sunnützlicher` Menschen zu verhindern. Diejenigen Refugiés, die sich in Torgau bereits vorgestellt hatten, waren arm, einige kamen gar $\mathrm{zu} \mathrm{FuB}$ aus Halle, andere, die mit Wagen kamen, wollten gleich die Reisekosten erstattet haben, die meisten forderten einen Vorschuß auf ihre vorhabenden Manufakturen, und übrigens [hätten sie] auf die aller menagirlichste Arth gelebt. Wenn es aber um wohlhabende Migranten, Capitalisten ging, änderte sich die Haltung, denn zu einem früheren Zeitpunkt war eine Ansiedlung von Refugiés in Torgau gescheitert, obgleich es lauter Capitalisten gewesen seien, um deren Einnehmung inständig ersucht worden war $^{22}$. Man verlangte also vom Landesherrn, wenn er denn schon Einwanderer verordnete, eine selektive Zuweisung von snützlichen، Hugenotten; die Armen und Bedürftigen wollte niemand, und eine autarke französische Gruppe innerhalb der Stadt wehrten die Torgauer Bürger wegen der erwarteten Konflikte ab.

Dasselbe Argument sollte wiederholt auftauchen und hatte schon in der Debatte um Leipzig Anfang des 18. Jahrhunderts eine Rolle gespielt: Durch die Zulassung des exercitium religionis für die Reformierten sei kein merklicher Aufschwung des Commerciums zu erhoffen; ebensowenig sei abzusehen, aus welchen Orten

große Capitalisten und solche Leuthe die sonderliche Handlung treiben sich anhero begeben möchten, [denn] die aus Franckreich Refugirten, so von ansehnlichen Vermögen gewesen, haben sich meistens in Engell- oder Holland, zum Theil auch in deren Chur-Brandenburg[ischen] Landen wesentlich niedergelaßen ${ }^{23}$.

Im Jahr 1713 machte Friedrich August I. einen neuen Versuch und brachte die Städte Meißen, wiederum Torgau und Oschatz als Ansiedlungsorte ins Ge-

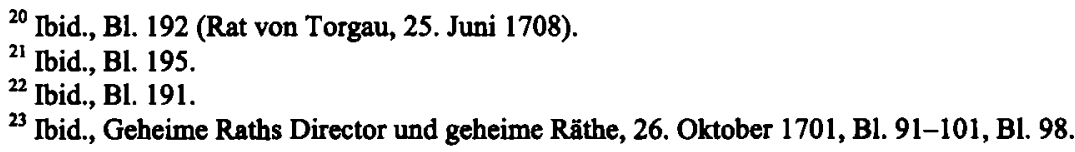


spräch $^{24}$. Diese neuerlichen Anstrengungen zeichnen sich im Vergleich zu den früheren Unternehmungen dadurch aus, daß jetzt die bisher am weitesten reichenden Zugeständnisse an die französischen Reformierten erwogen wurden.

Am 25. Februar 1713 war der preußische König Friedrich I. verstorben. Der sächsische Herrscher witterte nun eine Gelegenheit, unter der Hand eine Politik der Abwerbung preußischer Untertanen zu betreiben. So erging am 8. Juni 1713 von Friedrich August I. aus Warschau eine umfangreiche Instruktion für den Conseiller Le Coq am Berliner Hof ${ }^{25}$. Der sächsische Geheime Rat und Kabinetts-Sekretär Jacques Le Coq, Mitglied der Dresdner reformierten Gemeinde, sollte die umlaufenden Gerüchte über die Unzufriedenheit der Hugenotten in Brandenburg nach dem Tod des preußischen Königs prüfen und ihre Bereitschaft ausloten, nach Sachsen umzusiedeln. Sollte er auf solche unzufriedenen Refugiés stoßen, hatte er deren état, charactere, condition et facultez zu prüfen,

pour savoir s'ils sont veritablement en etat d'entreprendre quelques commerces et etablir des manufactures, qui puissent tourner au profit de Sa Majesté et de ses Etats, l'intention de Sa Majesté n'etant point d'introduire dans ses Etats des gens qui ne seroient pas en etat de gagner leur vie par leur industrie et par leur labeur et d'y faire fleurir le negoce; et qui ne seroient attirez que par l'esperance d'y avoir des pensions, ou jouir d'autres avantages pour leur commodité particulière - sans qu'il en revient aucun bien au pais ${ }^{26}$.

Erwünscht waren bons marchands, banquiers, manufacturiers et artisans. Le Coq besaß Vollmacht, ihnen die Protektion des sächsischen Kurfürsten und diverse Privilegien anzubieten. Darunter zählten neben der Befreiung von allen Lasten und Landessteuern über eine Reihe von Jahren l'exercice libre ${ }^{27}$ de la Religion Reformée dans les Villes, qui leur seront assignées. Deshalb sollte der Abgesandte besonderes Augenmerk auch auf die Anwerbung von angesehenen Pastoren legen. Diese Zugeständnisse, die hier unter der Hand für Sachsen offeriert wurden, gingen im ganzen über den tatsächlichen rechtlichen Status der Leipziger Reformierten deutlich hinaus.

Um zu diesem Zeitpunkt überhaupt noch unzufriedene Refugiés - Kapitalisten! - mit den erwünschten Qualitäten aus dem Nachbarland nach Sachsen zu locken, konnte der Kurfürst schlechterdings nicht weniger anbieten, als sie dort schon besaßen ${ }^{28}$. Womöglich deshalb erscheinen die Angebote, zu denen Le Coq in Berlin autorisiert war, beinahe übertrieben willfährig:

\footnotetext{
${ }^{24}$ SHStAD (wie Anm. 3), Instruction pour le Conseiller Le Coq à la Cour de Berlin, Art. 8.

${ }^{25}$ Ibid., Loc. 3351, S. 23-25.

${ }^{26}$ Ibid., Artikel 3.

${ }^{27}$ Allerdings mit dem Zusatz tel que les Francois Reformez en jouissent actuellement à Leipzig, so daß im Grunde wohl ein exercitium privatim gemeint war.

${ }^{28}$ Es blieben allerdings auch Fragen offen, etwa das Recht auf Kirchenbau, die Frage der kirchlichen Selbständigkeit, die Regelung von Rechtssachen u.ä.
} 
- in der freien Religionsausübung dürfe niemand sie stören oder beeinträchtigen;

- den Pastoren wurden staatliche Pensionen versprochen, von denen sie commodement leben können, d.h. ihre Bezahlung fiele nicht zu Lasten der Gemeinde;

- die Steuerbefreiungen und sonstigen Privilegien sollten für eine Frist gelten, die die reformierten Franzosen selbst bestimmen durften, allerdings erwartete der Kurfürst propositions raisonnables;

- die vorgeschlagenen Ansiedlungsstädte Meißen, Torgau und Oschatz galten nicht als obligatorisch, sondern über die Wahl eines Ortes sollten die Reformierten selbst entscheiden.

Die englische Königin sollte als Garantin für die zugesicherten Bedingungen eintreten. Dies sollte der avisierten Zielgruppe Sicherheit einflößen und die Tatsache kompensieren, daß Friedrich August sein Edikt über die Aufnahme französischer Refugiés nicht veröffentlichen wollte, pour ne point donner sujet de mecontentement à la Cour de Prusse qui ne manquera pas de prendre $d$ 'abord des mesures efficaces pour empecher les dits etablissements ${ }^{29}$.

Das beschriebene Projekt der Abwerbung von Untertanen des Brandenburger Vetters lief unter dem Siegel höchster Geheimhaltung. Dabei war die wechselseitige Abwerbung von Arbeitskräften durchaus eine gängige Praxis. Im selben Jahr 1713 wurden sächsische Arbeitskräfte von der Meißner Porzellanmanufaktur abgeworben, nachdem schon früher Handwerker und Manufakturarbeiter abgeworben worden waren ${ }^{30}$. Das ging so weit, da $\beta$ man preußische Emissäre in Kursachsen verhaften ließ. Das Projekt der Hugenottenabwerbung bildet also auch ein Element im Zoll- und Wirtschaftskrieg zwischen Sachsen und Brandenburg-Preußen.

Der Geheime Rat Le Coq stieß in Berlin auf den Tapissier Jean Pierre Mercier, der bereit war, mit seinen Arbeitern nach Sachsen zu kommen und nur für den König zu arbeiten. Die Idee nahm Gestalt an, in einer Dresdner Vorstadt eine Tapisseriemanufaktur einzurichten. 1714 war Mercier tatsächlich in Dresden $^{31}$ : Als inspecteur des tapisseries betrieb er eine Tapisseriemanufaktur vor dem Pirnaischen Tor und erhielt vom Hof eine jährliche Pension von 400 Talern $^{32}$. Am kursächsischen Hof waren außer diesem Gobelinfabrikanten

${ }^{29}$ SHStAD, Instruction (wie Anm. 24), Art. 11.

${ }^{30}$ Gross, Geschichte Sachsens (wie Anm. 1), S. 140f.

${ }^{31}$ Mercier wurde Mitglied der Dresdner reformierten Gemeinde. 1716 trat er in das Konsistorium der reformierten Gemeinde ein und gehörte diesem bis zu seinem Tode (1729) an; Gustav ROSENHAGEN, Geschichte der evangelisch-reformierten Gemeinde in Dresden 1689-1835, Dresden 1934, S. 74.

${ }^{32}$ Karl CzOK, August der Starke und Kursachsen, Leipzig 1988, S. 211. Die Geschichte der Hugenotten in Dresden wird in meiner in Arbeit befindlichen Monographie über Hugenotten in Sachsen 1700-1850 behandelt. Über die Dresdner hugenottischen Handschuhfabrikanten vgl. knapp Katharina MIDDELL, Hugenotten in der Wirtschaft, in: Passage Frankreich- 
(1726) weitere französische Reformierte beschäftigt, darunter die Architekten Raymond Leplat und Zacharias Longuelune, der Maler Louis de Silvestre und der Bildhauer François Coudray, die allesamt kunsthistorisch bedeutsam wurden.

Insgesamt mißlang jedoch die Mission Le Coqs. Die Zusicherung der freien Religionsausübung und weiterer Privilegien aus dem Jahr 1713 wurde ebensowenig verwirklicht. Wäre es dazu gekommen, hätte Sachsen gleichsam sein inoffizielles Edikt von Potsdam nachgeholt.

Der Auftrag Le Coqs wirft allerdings ein Licht auf die durchaus vorhandene Absicht Augusts des Starken, wirtschaftlich am Zufluß der Hugenotten zu partizipieren. Darin folgte seine Motivation der merkantilistischen Wirtschaftspolitik, wie sie alle deutschen Landesfürsten in dieser Zeit betrieben. Dieses bisher in der Forschung unberücksichtigte sächsische Ansiedlungsprojekt von 1713 manifestierte eine ebenso politische wie wirtschaftliche Absicht, aber von einer Einwanderungspolitik war es weit entfernt.

Es gibt mehrere Antworten auf die Frage, warum die Mission Le Coqs scheiterte. Erstens war der Zeitpunkt - 1713 - sehr spät gewählt, anderwärts bereits etablierte Hugenotten zu einem neuerlichen Umzug zu bewegen. Zweitens bremsten die gerade wieder aufgefrischten Beschwerden der Stände und der Geistlichkeit eine allein auf landesfürstliche Initiative zurückgehende Einladungsabsicht. Das dritte und wohl entscheidende Hemmnis war das strukturelle Dilemma eines katholischen Landesherrn in einem durch und durch lutherischen Land mit ungebrochener Herrschaft der lutherischen Orthodoxie. Sachsen genoß allerorten den Ruf, ein Hort konfessioneller Erstarrung zu sein. Wenn es so etwas wie ein kollektives sozial-religiöses Trauma gibt, dann war es die Episode des Kryptokalvinismus für Kursachsen während der sogenannten "Zweiten Reformation ${ }^{33}$. Der Leipziger Pastor Gabriel Dumont schrieb im Kontext des ersten Ansiedlungsprojekts für Torgau (1707) an den reformierten Haushofmeister des Königs:

Or un de ces obstacles [für die Niederlassung von Hugenotten] est assurement l'aversion que les Lutheriens tesmoignent contre les reformez, or on ne manquera pas de representer aux refugiez qui pourroient penser a venir s'establir en Saxe, que les Lutheriens du pais ont tou-

Sachsen. Kulturgeschichte einer Beziehung 1700 bis 2000, Katalog, Halle/Saale 2004, S. 70-73; DIES., »Refugirte« im Kernland der Reformation. Die Integration der Hugenotten in Sachsen, in: Sabine BENEKE, Hans OTTOMEYER ( $\mathrm{Hg}$.), Zuwanderungsland Deutschland. Die Hugenotten, Berlin, Wolfratshausen 2005, S. 73-80, hier S. 75.

${ }^{33}$ Thomas KLEIN, Der Kampf um die Zweite Reformation in Kursachsen 1586-1591, Köln, Graz 1962; Karlheinz BLASCHKE, Religion und Politik in Kursachsen 1586-1591, in: Heinz SCHILlNG (Hg.), Die reformierte Konfessionalisierung in Deutschland - Das Problem der "Zweiten Reformation«, Gütersloh 1986, S. 79-97. Blaschke zieht die problematische Schlußfolgerung, daß das Luthertum »der mitteldeutschen Volksart gemäßer« war als der Versuch der Einführung des Kalvinismus, der wintellektualistisch, rational, kühl und im ganzen `unsächsisch«« sei, so daß seinem Scheitern nicht nachzutrauern wäre (S. 96f.). 
jours esté extremement rigides contre nous, et qu'ils ne scauroient qu'avoir mille chagrins parmi un peuple aigri a tout moment par le zele indiscret de ces predicateurs, et de Theologiens outrez. Vous scavez que dans toute l'Europe on a ces idees des Lutheriens Saxons ${ }^{34}$.

Eine ähnliche Chance zur Aufnahme französischer Glaubensflüchtlinge ergab sich noch einmal während der Herrschaftszeit Friedrich Augusts II. (1733-1763), als im Frühjahr 1751 in einer weiteren Fluchtwelle Protestanten aus dem Languedoc flüchteten. Der sächsische Legationsrat in Hamburg, Gabriel von der Lith, berichtete darüber an die Geheime Kanzlei und hob hervor, $\mathrm{da}$ alle Flüchtlinge wohl gekleidet waren und anscheinend Gelegenheit gefunden [haben], ihre Baarschaften und andere transportable Mittel von einigen Werthe mit aus Frankreich heraus zu bringen ${ }^{35}$. Die Antwort der Geheimen Kanzlei an den Gesandten war eine direkte Aufforderung zur Abwerbung, wie seinerzeit an Le Coq in Berlin: Er sollte unter dergleichen Refugiés, Fabricanten, und Manufacturiers, sonderlich solche, da etwa noch mit einigem Vermögen versehen, oder die eine vorzügliche Geschicklichkeit besizen mit guter Art und Behutsamkeit veranlassen, daß sie ihren künftigen Aufenthalt in Sachsen nehmen ${ }^{36}$. Die Zusage von Schutz und Förderung erschien von der Lith jedoch zu unkonkret. Auf seine Nachfrage, worin sothane Beforderung eigentlich bestehen dörffte, ob und wie viele Freijahre oder welche sonstigen Vorteile er den französischen Flüchtlingen versprechen dürfte ${ }^{37}$, folgte Schweigen, und die Akte endet.

Zusammenfassend läßt sich zur Frage nach einer Einwanderungspolitik folgendes feststellen:

In Kursachsen führten Landesverfassung und Konfessionsstand und das unter Friedrich August I. prekäre Verhältnis zwischen katholischem Landesherrn und lutherischer Geistlichkeit bzw. den Ständen dazu, daß die hugenottische Migrationswelle im wesentlichen abgewehrt wurde. Die (mißglückten) Ausnahmen, die allein auf das Handeln des Kurfürsten und Königs zurückgingen, wurden oben beschrieben. Die Gegner einer Aufnahme von Reformierten von Amts wegen die Geheimen Räte, die Konsistorien, die Landstände, nicht zu vergessen die Theologischen Fakultäten Leipzig und Wittenberg, in den Städten die Räte und Bürgermeister - ließen eine landesfürstlich gesteuerte `Einwanderungspolitikı gar nicht erst aufkommen. Sie wollten zuerst den $\mathrm{Zu}$ zug von Kalvinisten überhaupt abwehren, und nachdem kleinere Gruppen dennoch eingewandert waren, ihnen die Ausübung ihres Gottesdienstes verweigern und eine Institutionalisierung als Gemeinde verhindern ${ }^{38}$. Für große

${ }^{34}$ Zit. nach KIRCHHOFF, Geschichte der Reformirten Gemeinde (wie Anm. 15), S. 231 f.

${ }^{35}$ SHStAD (wie Anm. 3), Loc. 5422, Bl. 1, 12. Mai 1751.

${ }^{36}$ Ibid., Bl. 2.

${ }^{37}$ Ibid., Bl. 3-4, Hamburg, 12. Juni 1751.

${ }^{38}$ Le grand consistoire de Dresde prétend que le nouveau recueil de cantiques allemands publiè par le pasteur Zollikofer, attribue à l'église une qualité qu'elle n'a pas, celle de Ge- 
Mißstimmung sorgte im übrigen auch der Unterschied im Gebrauch des Begriffs Konsistorium, das für die Reformierten etwas anderes bedeutete als für die lutherische Kirche.

Zudem wurde die Gewinnung von hugenottischen Fabricanten und Manufacturiers auch vom Landesherm zu spät und nicht nachdrücklich genug betrieben. Als König von Polen hatte Friedrich August andere Sorgen. Es blieb bei sporadischen Anläufen in Bezug auf die Förderung einzelner Städte. Die sächsische Wirtschaft äußerte kein ausgeprägtes Bedürfnis nach zahlreichen fremden Arbeitskräften, das Manufakturwesen nahm im 18. Jahrhundert auch ohne sie einen enormen Aufschwung ${ }^{39}$. Diejenigen französischen Manufakturisten aber, die einmal da waren, fielen unter die landesherrliche Wirtschaftspolitik, d.h. sie erhielten Zuschüsse und Prämien für ihre Manufakturen und verschiedene Gründungsbeihilfen. Da ihre Ansiedlung aber individuellen Entscheidungen folgte, lassen sie sich nicht auf irgendeine politische Intention zurückfuihren.

\section{3. »Freiwillige Niederlassung« von Hugenotten}

Für Kursachsen läßt sich nicht von einer gezielten Aufnahme von Hugenotten sprechen. Das wichtigste strukturelle Hindernis wurde auch benannt: die Erhaltung und Reinhaltung der Landesreligion. Unter der Fahne der Religion wurde vom Geheimen Rat und vom Oberkonsistorium eher eine Verhinderungspolitik betrieben. Eine Stadt aber, nämlich Leipzig, bot zugleich den wichtigsten strukturellen Anreiz für die Niederlassung von Reformierten, genauer gesagt von reformierten Kaufleuten. Die Leipziger Messe und die guten wirtschaftlichen Aussichten führten dazu, daß schon einige Jahre vor der Aufhebung des Edikts von Nantes schweizerische und französische Kaufleute hier ihren Wohnsitz nahmen. Es läßt sich nicht mehr klären, in welchem Maße religiöse Verfolgung und Flucht vor 1685 ein Motiv der Niederlassung war. Das wichtigste historische Buch der Leipziger reformierten Gemeinde, das 1804 als hommage [...] à la mémoire des premiers réfugiez geschrieben wurde, beginnt jedenfalls mit folgenden Worten:

Après la révocation de l'Edit de Nantes, en 1685, et même avant cette mémorable époque, quelques-uns des réfugiez sortis de France pour cause de religion, se transportèrent en

meinde; Archiv der Evangelisch-Reformierten Kirche Leipzig (= ARKL), Abrégé historique annuel de l'Eglise réformée de Leipzig, extraits des registres de cette église, Handschrift [1804], Eintrag 1766.

${ }^{39}$ Rudolf FORBERGER, Die Manufaktur in Sachsen vom Ende des 16. bis zum Anfang des 19. Jahrhunderts, Berlin 1958. 
Saxe: ceux d'entre-eux que le commerce avait attirés à Leipzig y trouvèrent plusieurs négocians Suisses et Genevois dont les établissemens, déjà tout formés, remontaient jusqu'à l'année $1670^{40}$.

Nachdem also ein Kern von reformierten Kaufleuten in Leipzig ansässig war und aus dem nahegelegenen preußischen Halle weiterer Zuzug erfolgte ${ }^{41}$, wurde auf Betreiben von 17 Familienhäuptern eine Gemeinde gegründet. Sie luden einen Prediger aus Genf ein, der im Dezember 1700 den ersten Gottesdienst hielt. Mehrere Bittschriften an den Kurfürsten und König und die Zahlung der beträchtlichen Summe von 7000 Reichstalern führten dazu, daß Friedrich August im Juli 1701 schließlich das exercitium religionis privatim erlaubte $^{42}$. Im Vergleich mit den Zusicherungen, die Le Coq für die Berliner Hugenotten in der Tasche hatte, bot diese beschränkte Kultusfreiheit wenig Anreiz. Im Verhältnis zum Widerstand gegen die Duldung der Reformierten, der sich noch über Jahre in einer Flut von Schriften aus Dresden und Leipzig an den Landesherrn ergoß, war dieses Zugeständnis allerdings viel.

Die 17 Gemeindegründer waren nicht die einzigen, wohl aber die reichsten Refugiés in Leipzig, die innerhalb sehr kurzer Zeit eine große Geldsumme aufbringen konnten ${ }^{43}$. Diese wohlhabenden reformierten Familienhäupter (chefs de famille contribuant à notre eglise) bezahlten alles: das Gehalt der Prediger, Kantoren, Küster und Schulmeister, die Kirchenutensilien, Miete, Ausstattung und Bauarbeiten an den Kirchenräumen usw. Die gesamte Gemeinde der Hugenotten zählte zur Jahrhundertmitte rund 500 Mitglieder. Zudem sorgte die Mobilität der Hugenotten im Refuge für Zuwanderung aus anderen deutschen Städten (für Leipzig nachweislich aus Halle, Magdeburg, Erlangen, Berlin) ${ }^{44}$. Noch im 18. Jahrhundert kamen auch aus Frankreich weitere Religionsverwandte hinzu.

Der juristische Status der reformierten Minderheit in der Stadt war der von sogenannten Schutzverwandten. Gegen ein jährliches Schutzgeld an die städti-

${ }^{40}$ ARKL, Abrégé historique (wie Anm. 38).

${ }^{41}$ Adolf ZAHN, Die Zöglinge Calvins in Halle an der Saale, Halle 1864; KIRCHHOFF, Geschichte der Reformirten Gemeinde (wie Anm. 15), S. 27-58; Martin GABRIEL, Die reformierten Gemeinden in Mitteldeutschland. Geschichte und Bekenntnisminderheit im 18. Jahrhundert und danach, Witten 1973, S. 51-53; Henri TolLIN, Geschichte der Französischen Colonie von Magdeburg, Bd. 2, Halle 1887, S. 21-80.

${ }^{42}$ KIRCHHOFF, Geschichte der Reformirten Gemeinde (wie Anm. 15); Paul WEINMEISTER, Beiträge zur Geschichte der evangelisch-reformierten Gemeinde zu Leipzig 1700-1900, Leipzig 1900.

${ }^{43}$ Einige Vergleichszahlen: Der Jahreslohn des Küsters wurde mit 16 Talern festgesetzt, der erste Pastor Butini aus Genf bekam 300 Taler im Jahr, die Gehälter der Pastoren stiegen bis zur Jahrhundertmitte auf 800 Taler jährlich (was im Vergleich zur staatlichen Besoldung der Prediger in Brandenburg sebr viel war). Und nun in wenigen Wochen $7000 \mathrm{Taler}$, mehr als das zwanzigfache Jahresgehalt des Predigers.

${ }^{4}$ Johannes HoHLFELD, Leipziger Geschlechter, Bd. 3: Die reformierte Bevölkerung Leipzigs 1700-1875, Leipzig 1939. 
sche Kasse genossen die Hugenotten ein dauerndes Bleiberecht. Politisch und zivilrechtlich waren sie bis 1811 benachteiligt. Sie durften keine öffentlichen Ämter bekleiden, kein Grundeigentum erwerben, waren steuerlich stärker belastet als die Bürger, hatten eingeschränkte Möglichkeiten der Berufswahl usw. Da ihnen aus Konfessionsgründen der Zugang zum Bürgerrecht verschlossen war, konnten sie in Leipzig und Sachsen keine whugenottischen« Wirtschaftszweige ansiedeln ${ }^{45}$, weil das städtische Bürgerrecht die Voraussetzung war, um in Zünfte und Innungen eintreten zu können. Handwerk und Gewerbe blieben den Hugenotten somit zwangsläufig verschlossen, und auch die Leipziger Universität konnte keinen Gewinn aus der Präsenz reformierter Intellektueller ziehen - während sie in Berlin bekanntlich eine herausragende Rolle spielten. Nur Kaufleute, und zwar Großkaufleute, durften Messe- und Transithandel treiben, weil allein der Engroshandel von zünftischen Regeln frei blieb ${ }^{46}$. Daraus resultierte eine besondere soziale Homogenität der Leipziger Hugenotten: Die Gemeinde wurde absolut dominiert von Kaufleuten, weshalb in den zeitgenössischen Dokumenten in Sachsen auch meistens von reformierten Kaufleuten die Rede ist.

Trotz aller Hindernisse und Nachteile des mehrfachen Minderheitenstatus konfessionell, "national«, juristisch, sprachlich - blieben diese freiwillig zugezogenen Hugenotten in Leipzig. Die wirtschaftliche Sicherheit im Verbund mit europaweiten Geschäfts- und Familienbeziehungen bewog sie zum Bleiben. Die anfängliche Abwehrhaltung gegenüber den wenigen Hugenotten ging in eine Art widerwillige Duldung über. Da sie mehrheitlich potente, zahlungskräftige Einwohner waren, nutzten sie der Stadt und trugen auch bei zum Aufschwung der Leipziger Messe zum zentralen deutschen Handelsplatz im Laufe des 18. Jahrhunderts ${ }^{47}$.

Die Integration ${ }^{48}$ der Hugenotten verlief als andauernder Prozeß über Jahrzehnte hinweg, in denen sich die allmähliche Akzeptanz der Reformierten, die Anerkennung der Differenz und die Überwindung der Distanz zwischen ihnen und Lutheranern im Alltagsleben entwickelte, bis ihnen schließlich im Jahr

${ }^{45}$ Über die soziale und berufliche Differenzierung der Französischen Kolonie in Berlin siehe z.B. Jürgen WILKE, Die Französische Kolonie in Berlin, in: Helga SCHULTZ, Berlin 16501800. Sozialgeschichte einer Residenz, Berlin 1987, S. 361-393; Stefi JERSCH-WENZEL, Juden und »Franzosen« in der Wirtschaft des Raumes Berlin/Brandenburg zur Zeit des Merkantilismus, Berlin 1978.

${ }^{46}$ Katharina MIDDELL, »En gros« und »en détail«. Konflikte um den Kleinhandel außer den Messen, in: Hartmut ZWAHR, Thomas TOPFSTEDT, Günter BENTELE (Hg.), Leipzigs Messen 1497-1997. Gestaltwandel - Umbrüche - Neubeginn, Köln, Weimar, Wien 1999 (Geschichte und Politik in Sachsen, 9/1), S. 231-242.

${ }^{47}$ Peter BEYER, Leipzigs Auseinandersetzungen mit Frankfurt am Main (1706-1726). Symptom des Aufstiegs zur führenden deutschen Messestadt, in: ZWAHR, TOPFSTEDT, BENTELE (Hg.), Leipzigs Messen (wie Anm. 46), Bd. 1, S. 193-204.

${ }^{48}$ Auf eine Diskussion der problematischen Aspekte des Integrationsbegriffs (ebenso "Assimilation« und »Akkulturation«) wird hier verzichtet. Vgl. auch Anm. 69. 
1811 die religiösen und bürgerlichen Rechte zugestanden wurden. Dieser Vorgang vollzog sich auf religiöser und auf gesellschaftlicher Ebene. Folgende Faktoren trieben die Integration voran bzw. manifestierten sie:

Die Reformierten respektierten die lutherischen Feiertage, sie versuchten, ihre Gottesdienste ohne öffentliches Aufsehen zu halten und vermieden absehbare Zwischenfälle. Schon 1702 beschlossen die Chefs de famille ou de Negoce, qu'on se conformeroit a la dicipline de France en touts les articles qui ce pourront observer par raport au pays dans lequel nous sommes et de la manière qu'elle s'observe dans les Eglises voysines du Brandebourg ${ }^{49}$. Das Leben inmitten einer dominierenden lutherischen Kultur zwang zu Kompromißbereitschaft. So lautete 1720 eines der Kriterien für die Wahl des neuen Predigers, qu'il fut tolerant a l'egard de Messrs. nos freres lutheriens ${ }^{50}$.

Die Anpassung an die Bedingungen vor Ort bedeutete auch eine partielle Distanzierung von den Regeln der französischen Kirchenordnung. Als das Konsistorium über die Zulässigkeit lutherischer Paten beriet, wurden die einschlägigen Beschlüsse der französischen Synoden geprüft. Man richtete sich aber am Ende nach der Praxis in Brandenburg, unter anderem mit der Begründung, que d'ailleurs nous sommes bien aise de faire connoitre a nos freres Luth: que nous sommes animés d'un Esprit de paix ${ }^{51}$. In der Frage der Stellvertretung eines abwesenden Paten löste man sich von der Bindungskraft der Beschlüsse von Montauban 1594, tant parce que les Eglises voisines n'en font point de difficulte, que parce que le refuge a si fort dispersé les familles qu'on ne pourroit guere observer l'article de Montauban a la rigueur ${ }^{52}$.

Einzelne Persönlichkeiten genossen weit über die Leipziger Gemeinde und über die reformierten Kreise hinaus besonderes Ansehen: Der erste deutsche Prediger Georg Joachim Zollikofer gelangte bei Gelehrten und Gebildeten zu überregionalem Ruf ${ }^{3}$. Die während seiner Amtszeit 1758-1788 wachsende Ausstrahlung des reformierten Gottesdienstes trug in Leipzig erheblich zur

${ }^{49}$ ARKL (wie Anm. 38), Registre des affaires (A), fol. 3r. Die Orthographie wurde beibehalten.

${ }^{50}$ Weiter: $L$ 'on convient aussi qu'il etoit necessaire qu'il eut de l'érudition \& qu'il entendit les deux langues. ARKL (wie Anm. 38), Delibérations I, 5. Dezember 1720.

${ }^{51}$ Deshalb nous sommes convenus unanimement qu'on pourroit se departir de cet arrest du synode de Charenton [1631] et recevoir les Lutheriens parrains et marraines sans exiger la dite condition. Ibid., Deliberations I, 14. Juli 1712.

52 Ibid.

${ }^{53}$ Siehe Katharina MidDELL, simmer unter der Herrschaft der Vernunft، - Der Prediger Georg Joachim Zollikofer als Aufklärer, in: Peter SCHÓTTLER, Patrice VEIT, Michael WERNER (Hg.), Plurales Deutschland - Allemagne Plurielle. Festschrift für Étienne François Mélanges Étienne François, Göttingen 1999, S. 179-191; Katharina MIDDELL, »... die größere Aufklärung gehöret also zu den Absichten Gottes«. Der Prediger Georg Joachim Zollikofer (1730-1788) und die Aufklärung in Leipzig, in: Hans-Jürgen SIEVERS (Hg.), In der Mitte der Stadt. Die evangelisch-reformierte Kirche zu Leipzig von der Einwanderung der Hugenotten bis zur Friedlichen Revolution, Leipzig 2000, S. 44-59. 
Überwindung der Distanz zwischen den Konfessionsgruppen bei. Dennoch ist bis in die zweite Jahrhunderthälfte die Besorgnis der städtischen Geistlichkeit, die zu wiederholten Anlässen nach Dresden schrieb, nicht zu übersehen.

Auf der gesellschaftlichen Ebene spielte das sbürgerliche` Verhalten der Reformierten eine von außen stets beobachtete Rolle. Sie achteten auf die Konformität mit den Landesgesetzen und nahmen teil an außergewöhnlichen Belastungen der Stadt in Kriegszeiten, namentlich im Siebenjährigen Krieg, während der Französischen Revolution und der napoleonischen Zeit. Sie betonten ihre Zugehörigkeit zum Untertanenverband und ihren "sächsischen $\mathrm{Pa}$ triotismus «.

Als weiteres Indiz der Integration und der informellen Emanzipation ist die Mitgliedschaft in den geselligen Vereinen und Sozietäten zu nennen. Dort spielte nicht die Glaubenszugehörigkeit eine Rolle, sondern die 'Ebenbürtigkeit،, um ein Kriterium aus der adligen Lebenswelt zu entlehnen. In den Logen, in Gesellschaften wie »Harmonie«, »Ressource«, Tanzgesellschaften u.ä. formte sich die anerkannte Zugehörigkeit zum städtischen Bürgertum durch adäquate Lebensführung, gleiche soziale und wirtschaftliche Interessen und lokale Bindungen $^{54}$. Familien- und Verwandtschaftsverhältnisse wirkten integrativ, auch sie wurden nach sozialen und ökonomischen Aspekten eingegangen ${ }^{55}$.

Die Hugenotten pflegten weiterhin kulturelle Praktiken, die das städtische Bürgertum konfessionsübergreifend kennzeichneten (Immobilien, Landhauskultur, Badereisen, Kunstbesitz), wobei sie auf den informellen Beistand von lutherischen Geschäftsfreunden bei der Überwindung der juristischen Schranken angewiesen waren. So dokumentiert der Vorgang des Grundstückskaufs, der nur als "Lehnskauf « möglich war - die Reformierten brauchten als formalrechtlich auftretenden Käufer einen sogenannten Lehnsträger, der das Leipziger Bürgerrecht haben und folglich lutherisch sein mußte - die bereitwillige Unterstützung durch lutherische Kaufleute. David Antoine Duvigneau hatte sein Landhaus in Connewitz unter dem Namen des Kaufmanns Johann Gotthold Leßing erworben, Marc Antoine Dufour sein ebenfalls in diesem beliebten Vorort gelegenes Landhaus unter dem Namen des Baumeisters Johann Adolph Richter gekauft ${ }^{56}$. Jacques Marc Antoine Dufour hielt am 27. Juni 1795 explizit fest, daß er sein prachtvolles Stadthaus aus der Ursache weil ich Reformierter Religion bin nicht in Lehn nehmen konnte; als Käufer des Hauses

\footnotetext{
${ }^{54}$ Vgl. Katharina MIDDELL, Leipziger Sozietäten im 18. Jahrhundert. Die Bedeutung der Soziabilität für die kulturelle Integration von Minderheiten, in: Neues Archiv für sächsische Geschichte 69 (1998), S. 125-157.

${ }^{\text {s5 }}$ DIES., Hugenotten in Leipzig. Streifzüge durch Alltag und Kultur, Leipzig 1998, S. 111-136.

${ }^{36}$ Sächsisches Staatsarchiv Leipzig, Amt Leipzig, Nr. 6298, Testament von David Antoine Duvigneau; ibid., Bankhaus Frege \& Co., Nr. 221, Bl. 35b. Siehe auch Katharina MiDDELL, „Wir sind also hiesige Handels-Leute, gleichwie andere hiesige Bürger...«. Die französischen Kaufleute in Leipzig, in: SIEVERS (Hg.), In der Mitte der Stadt (wie Anm. 53), S. 25-43, bes. S. 37-39.
} 
war der Leipziger Bankier und Freund Christian Gottlob Frege aufgetreten. Das Schriftstück von Dufour endet:

Schließlich wünsche ich allen Bewohnern dieses Hauses Glück und Segen, wünsche daß es meiner Vaterstadt beständig wohl gehen möge, und daß zu der Zeit, da dieses gefunden werden wird, es denen Glaubensgenossen aller Religionen gestattet werde, Grundstücken auf ihren Namen zu besitzen, ohne genöthiget zu seyn sich zum lutherischen Glauben zu bekennen ${ }^{57}$.

Die selbst gewünschte Integration der Hugenotten und ihre Akzeptanz zeigte sich im politischen Engagement in der Zeit der napoleonischen Herrschaft: Einige Reformierte übernahmen eine exponierte Vermittlerrolle zwischen den französischen Militär- und Zivilbehörden und der Stadt Leipzig ${ }^{58}$, namentlich Jacques Ferdinand Dufour, der für seine Dienste 1816 unter Verleihung des Namens Freiherr Dufour von Feronce in den sächsischen Adelsstand erhoben wurde.

Um 1800 belegt die Aufnahme von Hugenottennachfahren in verschiedene städtische und bürgerliche Ehrenämter den Abschluß ihrer Eingliederung in die Gesellschaft. Die Wahl in die Körperschaften der Kaufmannschaft, die sich lange gegen die »fremden Kaufleute« gewehrt hatten, kann als Indiz für die formelle Integration in die wichtigste soziale Gruppe der Stadt genommen werden. 1795 wurde Gabriel Philippe Duvigneau als Vertreter der Leipziger Kaufmannschaft zum Handelsdeputierten gewählt, 1807 folgte Jacques Ferdinand Dufour, 1811 Jacques Henri Thieriot ${ }^{59}$. Ferdinand Dufour war 1803 in das Direktorium der Leipziger Armenanstalt aufgenommen worden, was den Vater und Verfasser des »Abrégé historique« zu der Eintragung veranlaßte: C'est le premier exemple de cette nature dans notre église ${ }^{60}$. Derselbe Ferdinand Dufour war angesichts seiner Verdienste um Stadt und Land während der napoleonischen Zeit im Juni 1812 sogar zur Wahl in den Magistrat der Stadt vorgesehen, wäre dann der erste Reformierte überhaupt in der Stadtverwaltung geworden, doch er lehnte aus gesundheitlichen Gründen $a^{61} .1814$ wurde

${ }^{57}$ Stadtarchiv Leipzig, Nachlaß Dufour, Nr. 86. Jacques Marc Antoine Dufour hatte das Dokument in einer Vase auf dem Dach des Hauses deponiert. Hervorhebenswert ist in diesem Text die Verwendung der deutschen Sprache, wohl zum besseren Verständnis der Nachwelt, denn seine gesamte Familienkorrespondenz ist ansonsten französisch.

${ }^{58}$ Katharina MIDDELL, Leipzig und seine Franzosen. Die Réfugiés zwischen Sachsen und Frankreich am Ende des 18. Jahrhunderts und in der Napoleonzeit, in: Francia 26/2 (1999), S. 63-91, bes. S. 86-88.

59 Vgl. Stadtarchiv Leipzig, Handelsdeputierte, HA VI 1a, Bd. 15, Bl. 290, 17.2.1795; Bd. 16, S. 56, 9.4.1807.

${ }^{60}$ ARKL, Abrégé historique (wie Anm. 38), S. 131.

${ }^{61}$ Il remercie le sénat de cet honneur et le décline pour cause de santé. Ibid., S. 137. 
David Duvigneau in die Kramerinnung gewählt - dasselbe Organ, das einst seinen Vorfahren das Handelsgeschäft so erschwert hatt ${ }^{62}$.

Schließlich ist die Sprachentwicklung zu nennen, ein in der Forschung als 'klassisch angesehener Indikator für die Feststellung der hugenottischen Integration $^{63}$. Sie unterscheidet sich in Leipzig wohl kaum von den Prozessen in anderen Aufnahmeländern, was allgemein auch für die Mischehen als Indikatoren zu sagen wäre, für deren präzise Ermittlung jedoch die Quellenbasis nicht hinreicht. Ein schlagender Beweis für den Sprachwandel war die Berufung des ersten deutschen Predigers. Als Georg Joachim Zollikofer 1758 zum ersten Prediger neben dem französischen gewählt wurde - 1752 war die Wahl eines deutschen Predigers von den Familienoberhäuptern noch mehrheitlich abgelehnt worden -, stellte sich in der Diskussion der Familienoberhäupter heraus, daß von den französischen Gemeindegliedern nur einer nicht auch den deutschen Gottesdienst besuchen würde. Zum Zeitpunkt seiner Wahl am 15. Mai 1758 hatten 24 chefs de famille allerdings auch ausdrücklich festgehalten, la fondation de la colonie étant l'ouvrage des réfugiés français, toutes les écritures, \& notamment les registres ne cesseront d'être tenus dans la langue des fondateurs ${ }^{64}$. Sie bangten um ihre Identität, ihr kulturelles Gedächtnis, das in der gemeinsamen Geschichte einer Flucht bestand und durch die französische Sprache aufrecht erhalten und fortwährend reproduziert wurde.

In der dreißigjährigen Amtszeit des deutschen Predigers Georg Joachim Zollikofer vollzog sich in der Kirchengemeinde mehrheitlich der Wandel zur Deutschsprachigkeit. Nach seinem Tod (1788) wurde beschlossen, bei der Wahl des künftigen deutschen Pastors auf die Forderung nach Beherrschung der französischen Sprache zu verzichten. 1821 wurde das Französische als Kirchensprache abgeschafft, und nach dem Tod des Predigers Jean Louis Alexandre Dumas (1823) wurde auch kein französischer Prediger mehr eingestellt.

Zweisprachigkeit blieb aber bei denjenigen Kaufmannsfamilien erhalten, die Handelskontakte nach Frankreich unterhielten. Innerfamiliär läßt sich an Einzelfällen bis in die zwanziger Jahre des 19. Jahrhunderts demonstrieren, daß die jeweilige sprachliche Präferenz von deutsch oder französisch die Trennung von öffentlichem und privatem Raum markierte. Private Dokumente, Briefe, Tagebücher werden französisch geführt, obwohl darin sdeutsche Identitäten beschrieben werden.

${ }^{62}$ Auch dies zählte zu den Denkwürdigkeiten der Gemeindegeschichte: C'est le premier exemple d'un membre de notre Eglise admis à participer ici à gérer l'association des Merciers (Kramer-Innung). Ibid., S. 145.

${ }^{63}$ Mehrere Beiträge von Frédéric HARTWEG, zuletzt: Der Sprachwechsel im Berliner Refuge, in: BENEKE, OTTOMEYER, Zuwandenungsland Deutschland (wie Anm. 32), S. 121-126. Vgl. die Beiträge zum Thema Sprachwechsel in: Manuela BÖHM, Jens HÄSELER, Robert VIOLET (Hg.), Hugenotten zwischen Migration und Integration. Neue Forschungen zum Refuge in Berlin und Brandenburg, Berlin 2005. Vgl. auch Frédéric Hartweg und Franzsika Roosen in diesem Band. ${ }^{64}$ ARKL, Abrégé historique (wie Anm. 38), Eintrag 1758. 


\section{Zusammenfassung}

Die schrittweise Annäherung durch alltägliche Beziehungen und Kontakte, das Zusammenleben in einem überschaubaren Raum, besonders aber die soziale Äquivalenz der reformierten Kaufleute und der Familien des Leipziger Wirtschaftsbürgertums reduzierte allmählich die Bedeutung der religiösen Unterschiede. Mit der 1811 gewonnenen legalen Gleichberechtigung ${ }^{65}$ wurde ein Zustand festgeschrieben, der real bereits erreicht war. Das Mandat König Friedrich Augusts III. besagte:

daß den Reformirten Religionsverwandten in Allerhöchst dero Landen nunmehr freye und öffentliche Ausübung ihres Gottesdienstes, auch eben die bürgerlichen und politischen Rechte zugestanden werden sollen, welche dero Römisch Catholischen Glaubensverwandten durch das Mandat vom 10. Febr. 1807 eingeräumet worden sind, und welche die Augsburgischen Confessionsverwandten schon vorhin unverrückt genossen haben ${ }^{66}$.

Damit ging auch der Sonderstatus der reformierten Gemeinde verloren. Die Hugenotten in Leipzig (auch in Dresden) hatten nie um die Aufrechterhaltung eines Sonderstatus gekämpft, dazu waren es zu wenige. Sie wurden geleitet vom Wunsch nach Zugehörigkeit und Gleichberechtigung, und die erhielten sie nach mehr als 100 Jahren.

Verschiedene Einwanderungspolitiken der Obrigkeit spielten eine herausgehobene Rolle unter den äußeren Faktoren, die Integrationsvorgänge nach Migrationen bestimmen. Ein Vergleich der Maximen zweier Einwanderungspolitiken, nämlich Brandenburg-Preußens und derjenigen der Niederlande, speziell in der südafrikanischen Kolonie, verweist auf verschiedene politisch präformierte Wege der Integration bzw. der forcierten Assimilation. Den Hugenotten in Brandenburg-Preußen wurden großzügige Privilegien gewährt, eine eigenständige Administration eingeräumt, Pflege ihrer religiösen, kulturellen und sprachlichen Traditionen ermöglicht. Die Fürsten ordneten der intendierten Vorbildwirkung geschlossener Ansiedlung von Zuwanderern entsprechende Maßnahmen an, wie Friedrich II.:

Wenn fremde Familien angesetzt werden, so muß man sie nicht mit den einheimischen vermischen, sondern ganze Dörfer und Kolonien mitten unter dem groben und bunten Zeug anlegen,

${ }^{65}$ Mandat, die Gleichstellung der reformierten Religionsverwandten, hinsichtlich der öffentlichen Ausübung ihres Gottesdienstes, auch übrigen bürgerlichen und politischen Rechte, mit den römischkatholischen und Augsburgischen Confessionsverwandten betr., vom 18. März 1811, in: Dritte Fortsetzung des Codicis Augustei, worinnen die in dem Königreiche Sachsen ergangenen gesetzlichen Verordnungen vom Jahre 1801 bis zu der am 9. März 1818 angefangenen Gesetzessammlung, enthalten sind. Erste Abt., Dresden 1824, S. $17 \mathrm{f}$.

${ }^{66}$ Stadtarchiv Leipzig (wie Anm. 57), Bürgerbuch 1792-1819, Bl. $240 \mathrm{f}$. 
die ganz allein wohnen und ihre Nahrung und Gewerbe für sich treiben, damit das hiesige Volk um so besser siehet und gewahr wird, wie jene sich einrichten und wirtschaften ${ }^{67}$.

Dagegen versuchten die niederländischen Behörden in Südafrika, die Eigenständigkeit der hugenottischen Siedler stark einzuschränken, u.a. durch Anordnung einer gemischten Siedlungsweise - damit sie unserem Volk um so besser einverleibt werden - oder durch Verbot der französischen Sprache im Gottesdienst und in den Schulen, um dadurch diese Sprache mit der Zeit abzutöten und gleichsam zu verbannen ${ }^{68}$. Im ersten Fall vollzog sich die Integration der Hugenotten im Sinne des Hineinwachsens in die Aufnahmegesellschaft unter Beibehaltung von Teilen der eigenen kulturellen Identität ${ }^{69}$. Diese schlug sich in der mythisch verklärten historischen Erinnerung ein Jahrhundert nach der Einwanderung in der Betonung der zivilisatorischen Mission der Hugenotten für den Hohenzollernstaat nieder ${ }^{70}$. Im zweiten Fall ist eine rasche Assimilation im Sinne einer Angleichung der Zuwanderer an die Bevölkerung des Aufnahmelandes zu beobachten, wbei der die mitgebrachten Werte und Kulturmuster äußerlich weitgehend zurücktreten $\kappa^{71}$. Der politisch motivierte Assimilationsdruck auf die französischen Einwanderer in Südafrika führte zur »idealen Entstehung des weißen Südafrikaners « ${ }^{72}$.

Eine so deutliche politische Zielsetzung konnte sich in Sachsen in beiderlei Richtung nicht ausprägen. Trotz dieses Fehlens sei abschließend festgestellt, $\mathrm{da}$ sich auch in Leipzig und - in geringerem Maße - in Dresden kleine hugenottische Migrantengruppen ansiedelten, die eine Rolle als waktive Minder-

${ }^{67}$ Zitiert nach Frédéric HARTwEG, Die Hugenotten in Berlin: Eine Geschichte, die vor 300 Jahren begann, in: DERS., Steffi JERSCH-WENZEL (Hg.), Die Hugenotten und das Refuge. Deutschland und Europa, Berlin 1990, S. $22 f$.

${ }^{68}$ Jörg FISCH, Die Hugenotten am Kap der Guten Hoffnung oder Die ideale Entstehung des weißen Südafrikaners, in: Heinz DuchHARDT ( $\mathrm{Hg}$.), Der Exodus der Hugenotten. Die Aufhebung des Edikts von Nantes als europäisches Ereignis, Köln, Wien 1985, S. 89-120, hier S. $105 f$.

${ }^{69}$ Marita KRAUSS, Integration und Akkulturation. Eine methodische Annäherung an ein vielschichtiges Phänomen, in: DIES., Mathias BEER, Martin KINTZINGER ( $\mathbf{H g}$.), Migration und Integration. Aufnahme und Eingliederung im historischen Wandel, Stuttgart 1997, S. 11-25, hier S. 12.

${ }^{70}$ Vgl. Étienne FrançoIs, Die Traditions- und Legendenbildung im deutschen Refuge, in: DuchHardT (Hg.), Exodus (wie Anm. 68), S. 177-194; Frédéric HARTWEG, Hugenotten(tum) und Preußen(tum), in: Ingrid MITTENZWEI ( $\mathrm{Hg}$.), Hugenotten in BrandenburgPreußen, Berlin 1987, S. 313-352.

${ }^{71}$ KRAUSS, Integration und Akkulturation (wie Anm. 69), S. 15; FisCH, Hugenotten am Kap (wie Anm. 68), S. 101f.

72 „Die Hugenotten hatten eine bedeutende Wirkung auf die Entstehung des südafrikanischen Staates nicht dadurch, daß sie französische Traditionen beibehielten, sondern dedurch, daß sie zu besonders guten Südafrikanem wurden. Das Geheimnis ihres Erfolges liegt in der vollständigen Integration und Assimilation«. Ibid., S. 113. Obwohl fraglich bleibt, ob die erstrebte srückstandslose Assimilation tatsächlich so widerstandslos gemäß den politischen Intentionen erreicht wurde. 
heit« spielten ${ }^{73}$. Von ihrem französischen Ursprung zeugten im 19. Jahrhundert nur mehr klangvolle Namen.

\section{Résumé français}

Dans l'électorat de Saxe, les conditions d'établissement pour les huguenots ainsi que la relation entre les immigrants et la société d'accueil se distinguaient de ceux qui existaient dans les autres pays d'accueil en Allemagne.

Après une étude des conditions préalables existant en Saxe électorale, nous montrons que à part quelques signes sporadiques - il n'y avait pas de politique d'immigration concrète. Les autorités du pays et les municipalités tentaient d'éviter de toutes leurs forces l'admission des réfugiés qui voulaient s'y installer et de contoumer le peu de tentatives qu'il y avait de la part du prince électeur/roi Frédéric Auguste ["T/Auguste II. Pourtant, quelques petits groupes de migrants huguenots, soutenus par le souverain, s'installèrent à Leipzig et à Dresde. À long terme, ils jouaient le rôle d'une "minorité active«. Nous présentons plus en détail un projet d'établissement de 1713 qui, à l'aide d'activités diplomatiques secrètes, avait comme objectif de racoler les huguenots de Brandebourg-Prusse.

Dans la deuxième partie, nous étudions la formation de la communauté réformée et ses particularités sociales en priorité à partir de l'exemple de Leipzig. Pour les huguenots euxmêmes, la motivation conduisant a leur intégration civile résultait du statut juridique qu'on leur avait attribué (Schutzverwandte avec un droit de séjour, c'est-à-dire la condition d'habitants non bourgeois d'une ville, mais qui jouissent cependant de sa protection). Ce statut impliquait une multitude de conséquences politiques, sociales et économiques. À la différence de la polémique du clergé de la ville, celle de la bourgeoisie était surtout dirigée contre le patriotisme prétendu des »commerçants français« et voilait ainsi de temps à autre la peur considérable d'une concurrence économique.

En conclusion, nous abordons les divers facteurs qui, au fil du XVW" siècle, contribuèrent à la suppression successive du sentiment d'étrangeté et de distance et qui facilitèrent l'intégration sociale des huguenots jusqu'en 1811 - date à laquelle les huguenots obtinrent l'égalité des droits civils et religieux. Malgré l'ambivalence historique, on doit constater que la minorité huguenote constituait une expérience positive et à long terme apportait au pays d'accueil un surcroît culturel.

${ }^{73}$ Serge Moscovici, Psychologie des minorités actives, Paris 1979. 